\title{
Iot Integrated Industrial Automation for Wsn Applications
}

\author{
Ashwini Gona, Anil Kumar Gona
}

\begin{abstract}
To collect and analyze the data for physical parameter analysis in various applications such as Industrial monitoring wireless sensor network has been deployed in our research design. In this design, ZigBee based observing framework is developed with Internet of Things, using temperature/humidity sensors, moisture sensor, Gas sensor and water level sensor. Web of Things (IoT) has drawn tons of consideration and is required to be favorable to various application zones like modern WSN frameworks, and natural frameworks for better information obtaining for IoT portrayal. A sensor based information obtaining framework is a basic part for industrial automotive control frameworks. In this research the main concern is to establish the connectivity and computing capability to all the sensors using Arduino and ARM cortex. Proposing a novel method to plan a reconfigurable information securing framework for modern sensors, in which Arduino and ARM Cortex has been adopted as the core controllers. This system can read the real time data with high speed in parallel because here both dynamic and static system reconfigurations are used. Based on the ZigBee and internet protocol, brilliant Industry framework was planned with the execution of related programming and equipment which can associate the different units together and give a brought together interface to clients to communicate with the observing square. Some fundamental highlights are recorded, for example, smart sensor interface, lighting control, Fan control, Thermostat control, vibration monitoring, remote control, smart energy, security and safety.
\end{abstract}

Keywords: ARM Cortex, Internet of Things (IoT), WSN, Sensor Interface.

\section{INTRODUCTION}

Industrial automation is a method for automatic control of industrial appliances for user convenience. This technology saves energy by proper utilization of the devices according to the user strict requirement and it makes manufacturing and production easier for the user. Controlling can be as simple as controlling lights with a remote or as unpredictable as setting up a system of things in the industrial that can be customized utilizing a centralized microcontroller.

Apart from algorithmic automation, the user can control the devices to fulfill his/her personal requirements. For this, a system of machines and sensors needs to interact with one another to settle on decisions for required activity.

The proposed work provides an innovative design for cost-effective industrial computerization framework, first examining the general plan contemplations that ought to be assessed before beginning, followed by the implementation of design using Arduino and ARM Cortex.

Revised Manuscript Received on September 14, 2019.

Ashwini Gona, Asst.Professor, Dept Of Ece, Anurag Group Of Institutions, Hyderabad, Telangana, India.(Email: ashwiniece@cvsr.ac.in)

Anil Kumar Gona, Asst.Professor, Dept Of Ece, Anurag Group Of Institutions, Hyderabad, Telangana, India.(Email: anilkumarece@cvsr.ac.in)

\section{LITERATURE SURVEY}

\section{A. RELATED WORK}

With rapid advancement in IoT, most of the manufacturers are devoted for the examination of interfacing the multi sensor procurement gear [8]. There are numerous information procurement multiple interface equipment's are available on the market with mature technologies. To the changing IoT environment Interface devices are not easily adaptable [9] because the working style of these interface devices is specialized. Simultaneously, these universal information procurement interface gadgets are regularly confined in physical properties of sensors, for example, associate number, sampling rate, and signal types.. On other ways, CPLD/FPGA which is a [10], [11], enables us improve the real time performance of the system with parallel acquisition of multi sensor data [12]. At present, CPLD/FPGA has become more popular than MCU for data acquisition from multiple sensors in IoT condition. In any case, in IoT condition, the association of different industrial WSNs makes the framework complex because of assorted sensors. Each sensor has its own requirement for data acquisition and also users require different types of sensors for their own applications [13]. As a result, it brings many challenges to the researchers because it require very complex and bulky sensor driver code and information accumulation strategies for every single sensor which is recently associated with interface gadget [14]-[16]. For the clear study on industrial WSN applications, the key role is done by the sensor data acquisition device [17]. The IEEE Electronic Engineering Association has similarly pushed IEEE1451 smart transducer (STIM) interface standard show suite for the future progression of sensors in order to systematize a wide extent of Intelligent sensor Interfaces [18]. The convention presents the determinations from sensor interface definition to the information obtaining [19]. The STIM interface standard IEEE1451 empowers sensors to consequently look through system, and the STIM advances the improvement of modern WSN [20]. Sensors with the protocol standard are very costly these sensors lack in popularity for industrial WSN in IoT condition. Examples of Intelligent sensors open accessible and predictable with this standard are up 'til now confined [21]. For the capability of interfacing with different sensor typologies, some committed hardware interfaces dependent on the IEEE 1451 have been recently proposed [22]. These interface gadgets

Blue Eyes Intelligence Engineering

\& Sciences Publication 
are generally founded on moderately complex devoted electronic boards [23]-[24]. In IOT environment, a reconfigurable multi sensor information acquisition interface with incredible likeness and institutionalizing interface standard ought to be made. By focusing on the above work, this paper structures an IoT based Interactive Industrial remote system, and embedded data getting structure to appear nearby page. This plan presents numerous points of interest as portrayed underneath. Presently, Micro control unit (MCU) is utilized as the centre controller in standard information procurement interface device. Low cost and low control utilization are the benefits of MCU which makes it moderately simple to actualize. It plays out an endeavour by strategy for encroach upon, which makes these multi sensor acquiring interfaces genuinely parallel in social affair multi sensor data. Arduino and ARM Cortex is used as the inside controller to release the restriction on the general data acquisition interface, and recognize truly parallel getting of sensor data. Our endeavour not simply improved the sensor data aggregation profitability of modern WSN, yet what's more extended the application extent of the data acquiring interface gear in IoT condition. Besides, another structure procedure is proposed in this paper for multi sensor data verifying interface that can get connection and play for different sorts of sensors in IoT condition. In this paper, this plan exploit Arduino and ARM Cortex attributes, for example, high execution speed, adaptable association structure, IP configuration could reuse, and so on., which improves our gadget good in the field of modern WSN in IoT condition. The remainder of this paper is sorted out as pursues. The Microcontroller choice is introduced in Section III, and the Introduction of Hardware Architecture is described in Section IV. Schematic and working operation is presented in section V. The results for WSN \& Industrial automation is discussed in Section VI. Finally, we conclude our work in Section VII.

\section{B. SENSOR DATA ACQUISITION: DESIGN CONSIDERATIONS:}

The plan of information procurement framework for modern sensors regularly confronting the accompanying issues:

1) Universality of function: Data procurement framework is required to interface a wide assortment of sensors, which is a noteworthy test before the researchers. In any case, it is hard to actualize all the sensor conventions at the same time in a single system due to the lack of resources.

2) Universality of configuration: In practical industrial applications, it is very easy to make mistakes while configuring the files for units because different sensor combinations may be used by different functional units. So it has very complex download process.

3) Continuity of working: The basic requirement for industrial applications is the replacement of sensors, as an example, adding or reducing the sensors count, replacing some or all the sensors with similar types of sensors of other manufacturers' available in the market. In order to replace the sensor, we cannot poweroff and shutdown the entire system.

\section{REAL-TIME PERFORMANCE: THE REAL- TIME PERFORMANCE MUST BE VERY HIGH FOR INDUSTRIAL APPLICATIONS, WHILE THE READING CYCLE OF A TRADITIONAL SENSOR DEPENDS MORE ON THE EMBEDDED PROGRAMMING SKILLS.}

All these problems are solved by designing a reconfigurable data acquisition system using an Arduino \& ARM Cortex for industrial sensors which combines data acquisition technology with reconfiguration technology.

Our contributions are:

1) Proposing an architecture to modify the system to achieve the following functions:

a. The function-universality requirement can be met by time division multiplexing the resources by using reconfiguration technique such as switching the sensor protocol at any time.

b. In this system the sensor data acquisition function achieves the switching without any interruption to the other hardware equipment or changing the connections or powering off to the system. Such systems meet the complex requirements of sensor based industrial applications.

c. Here, both Arduino \& ARM Cortex are used simultaneously as main controllers for our design, so the parallel Interface of these controllers allows to collect the sensor data in a parallel.

\section{MICROCONTROLLER SELECTION}

ARM has developed quite a number of different processor products. The ARM processors are divided between the classic ARM processors and the newer Cortex processor product range. In addition, these processors are divided into three groups based on the application spaces:

Application Processors - Top of the line processors for portable figuring, advanced mobile phone, servers, and so on. These processors keep running at higher clock recurrence (over $1 \mathrm{GHz}$ ), and support Memory Management Unit (MMU), which is required for full highlight OS, for example, Linux, Android, MS Windows and mobile OSs. If you are planning to develop a product that requires one of these OSs, you need to use an application processor.

Real-time Processors - These are superior processors for continuous applications, for example, hard disk controller, automotive power train and base band control in remote correspondences. The greater part of these processors don't have MMU, and for the most part have Memory Protection Unit (MPU), store, and other memory highlights intended for industrial applications. They can keep running at a genuinely high clock recurrence (for example $200 \mathrm{MHz}$ to $>1 \mathrm{GHz}$ ) and have exceptionally low reaction dormancy. In spite of the fact that these processors can't run full forms of Linux or Windows, there are a lot of Real Time Operating Systems (RTOS) that can be utilized with these processors.

Microcontroller Processors - These processors are usually designed to have a much lower silicon region, and much high-vitality productivity. Normally, they have shorter 
pipeline, and for the most part lower greatest recurrence running at over $200 \mathrm{MHz}$. At the same time, the newer Cortex-M processor family is designed to be very easy to use; therefore, they are very popular in the microcontroller.

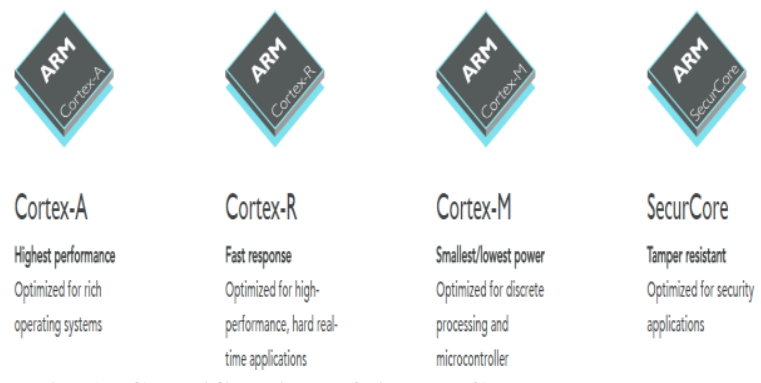

Fig 1: Classification of ARM Cortex processors

The ARM Cortex-M series, STM32F030x4/x6/x8/xC microcontrollers are structured with the elite ARM CortexM0 32-piece RISC center which is working at a $48 \mathrm{MHz}$ recurrence, 256 Kbytes of implanted blaze recollections with rapid and up to 32 Kbytes of SRAM and wide scope of upgraded peripherals and I/Os. ARM Cortex M0 offer standard correspondence interfaces two I2Cs, 2 SPIs and 6 Universal Synchronous Asynchronous Receiver Transmitters, one 12-piece ADC, seven broadly useful 16bit timers and a propelled control PWM clock. The gadgets incorporated into STM32F030x4/x6/x8/xC microcontrollers in four unique bundles running from 20 pins to 64 pins. These highlights make the STM32F030x4/x6/x8/xC microcontrollers appropriate for a wide scope of utilizations, for example, application control and UIs, handheld gear, $\mathrm{A} / \mathrm{V}$ collectors and advanced TV, gaming and GPS stages, mechanical applications, video radios, and HVACs, PC peripherals, PLCs, inverters, printers, scanners, caution frameworks,.

STM32F030x4/x6/x8/xC microcontrollers gives three low-control modes to decrease the power utilization. To accomplish the best bargain between low control utilization, short start up time and accessible wakeup sources:

- Sleep mode: In Sleep mode, just the processor stops its working though every one of the peripherals proceed to work and can awaken the processor when an interfere with/occasion happens.

-Stop mode: In Stop mode low control utilization is accomplished while holding the contents of SRAM and registers.
- Standby mode: Standby mode is utilized to accomplish the least control utilization while the interior voltage controller is turned off with the goal that the whole $1.8 \mathrm{~V}$ area is powered off.

\section{THE INTRODUCTION OF THE HARDWARE ARCHITECTURE}

A centralized architecture for Industrial WSN scenario is depicted in Fig 2 with sensor nodes connected to the network manager through Arduino. The gathered data from nodes is communicated to the network manager which in turn communicates this data to the process controller. The network manager controls and manages the sensor nodes and process controller. The WSN is used as a part of the control loop in the control automation segment of the industry, which has given rise to new features and these are referred as wireless sensor and actuator networks (WSAN). The industrial operating units like valve, thermostat, light, Fan \& heater can be controlled using process controllers through the commands given to the network manager via internet. Various wireless standards such as ZigBee are proposed to be used in Industrial WSN.

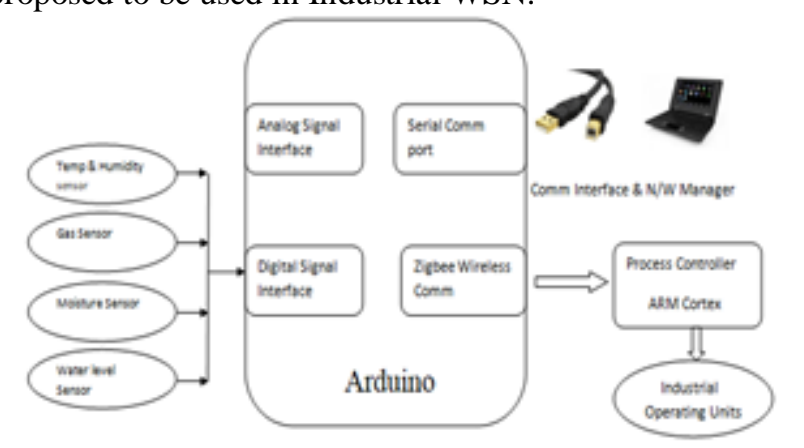

Fig 2: Architecture for the design of reconfigurable smart sensor interface design

\section{SCHEMATIC AND WORKING OPERATION}

According to the structure of reconfigurable smart sensor interface, it consists of Arduino \& communication circuit for providing an interface from USB to serial port of a laptop, high-speed 6-channel ADC, LED indicator, analog interface on Arduino which can connect six different sensors. This reconfigurable structure of smart sensor interface can handle up to six analog signals and 13 digital signals. In this paper, five independent sensors are interfaced namely DHT11 temperature sensor, humidity sensor, Moisture sensor, Gas sensor and water level sensor. Fig 3 shows the hardware schematic diagram of Arduino with sensors interfacings. 


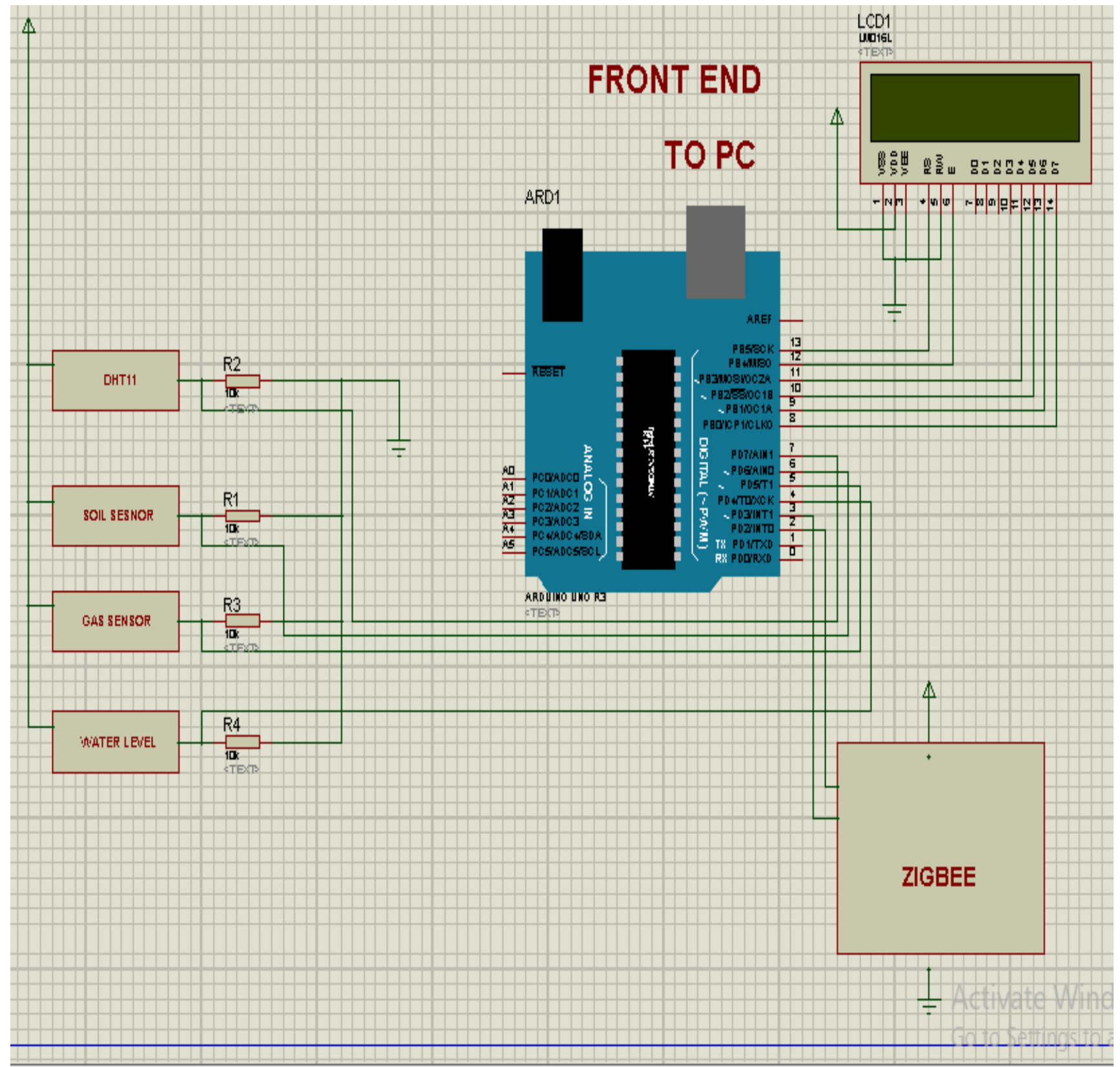

Fig 3: Schematic of sensor interfacing with Arduino

the Arduino board via software serial communication interface. When the load Control instructions are received

Apart from the basic sensor data acquisition, the hardware system can also send/receive data to/from the network manager via USB serial port and also to the process controller via a wireless communication module such as ZigBee. A ZigBee wireless module can be connected with by the process controller, the network manager acts as a wireless data transceiver node. The running status of the peripheral devices can be controlled with the corresponding data communication function. 


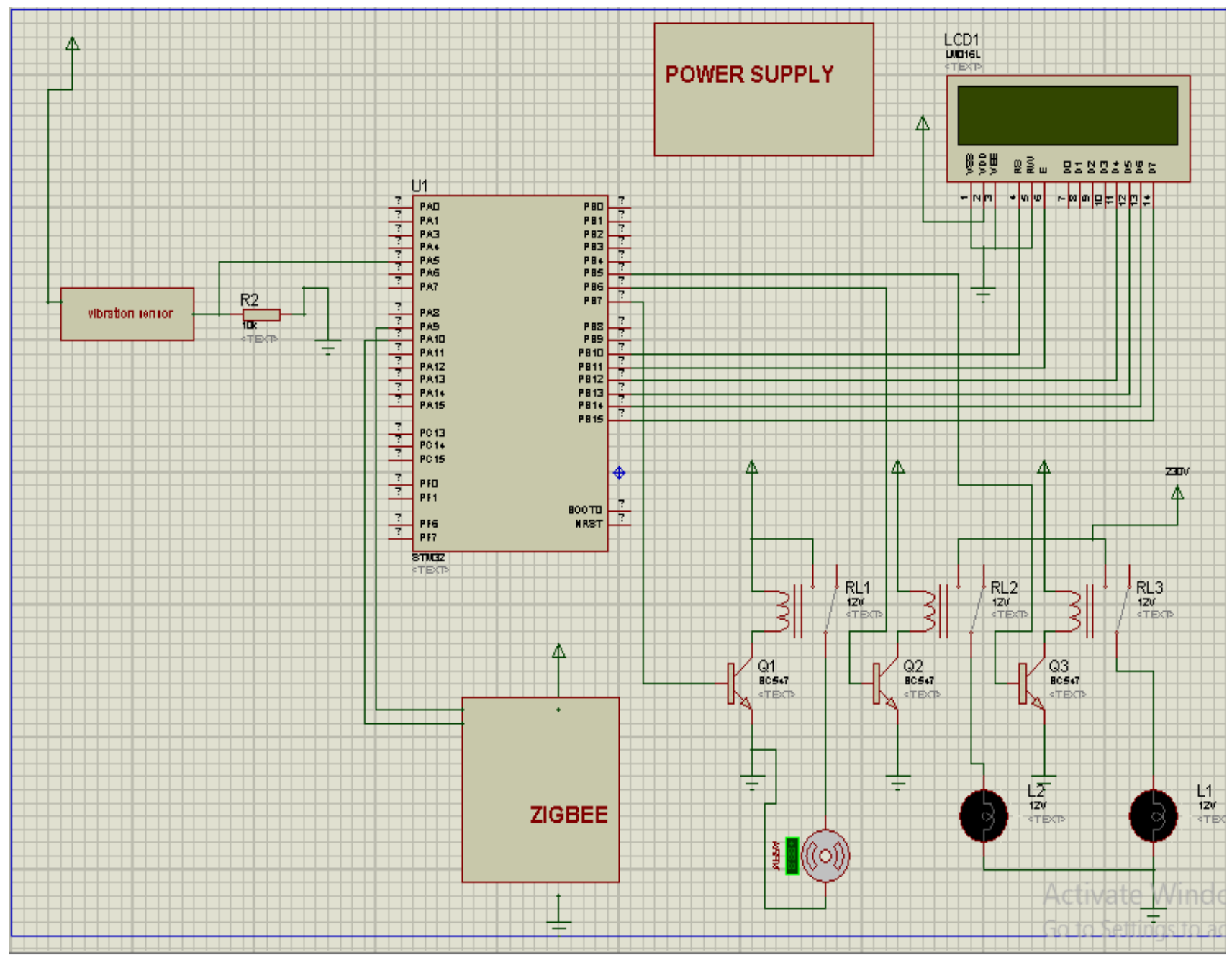

Fig 4: Schematic of Industrial automation using process controller ARM Cortex.

VII. RESULTS

The publisher could not be verified. Are you sure you want to run this software?

Name: C:IUsers\KANTHI\Desktop\Serial to server(1).exe

Publisher: Unknown Publisher

Type: Application

From: C:IUsers\KANTHI\Desktop (Serial to server(1).exe

Run

Cancel

$\checkmark$ Always ask before opening this file

Fig 5:Run the windows Form application

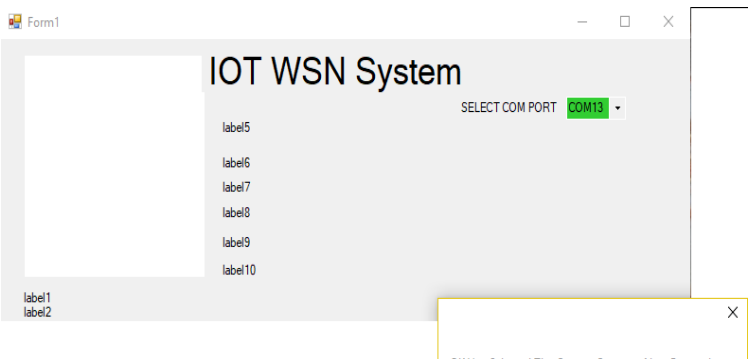

Fig6: Selecting the COM Port13

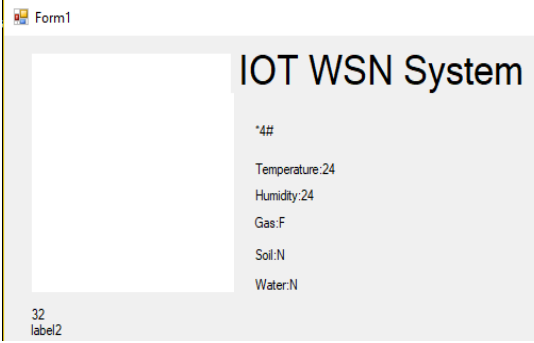

Fig 7:Sensor values updation to the Network Manager

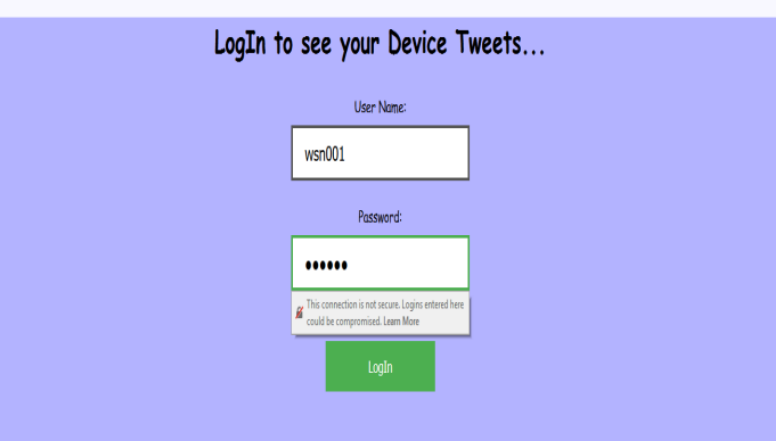

Fig 8: Login to the IoT web Server

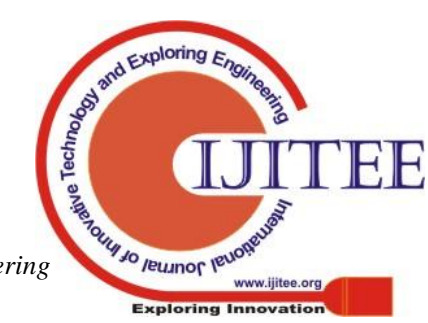




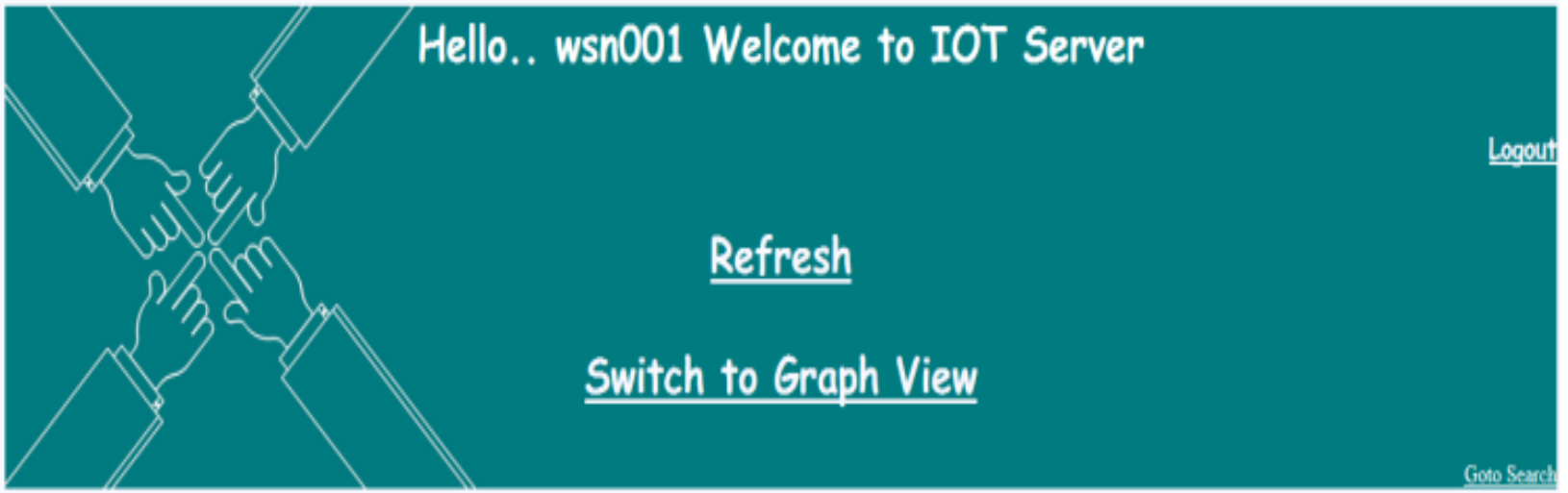

Poge 1 of 19 Next

\begin{tabular}{|c|c|c|c|c|c|c|c|}
\hline SNo & Temperature & Humidity & GAS & SoIL & WATER & \multicolumn{2}{|c|}{ Date } \\
\hline 1 & 24 & 78 & $F$ & $\mathrm{~N}$ & $\mathrm{~N}$ & & \\
\hline 2 & 24 & 78 & & F & $\mathrm{N}$ & $\mathrm{N}$ & $2017-11-05$ 08:58:46 \\
\hline 3 & 24 & 76 & & $\mathrm{~F}$ & $\mathrm{~N}$ & $\mathrm{~N}$ & 2017-11-05 08:58:19 \\
\hline 4 & 24 & 76 & & $\mathrm{~F}$ & $\mathrm{~N}$ & $\mathrm{~N}$ & $2017-11-05$ 08:57:53 \\
\hline 5 & 24 & 76 & & $\mathrm{~F}$ & $\mathrm{~N}$ & $\mathrm{~N}$ & $2017-11-0508: 57: 26$ \\
\hline 6 & 24 & 78 & & $\mathrm{~F}$ & $\mathrm{~N}$ & $\mathrm{~N}$ & 2017-11-05 08:57:00 \\
\hline 7 & 26 & 79 & & $\mathrm{~F}$ & $\mathrm{~F}$ & $\mathrm{~N}$ & $2017-10-2607: 40: 40$ \\
\hline 8 & 26 & 79 & & $\mathrm{~F}$ & $\mathrm{~F}$ & $\mathrm{~N}$ & $2017-10-2607: 40: 17$ \\
\hline 9 & 26 & 79 & & $\mathrm{~F}$ & $\mathrm{~F}$ & $\mathrm{~N}$ & $2017-10-2607: 39: 54$ \\
\hline 10 & 26 & 79 & & $\mathrm{~F}$ & $\mathrm{~F}$ & $\mathrm{~N}$ & $2017-10-2607: 39: 32$ \\
\hline 11 & 26 & 79 & & $\mathrm{~F}$ & $\mathrm{~F}$ & $\mathrm{~N}$ & $2017-10-2607: 39: 10$ \\
\hline 12 & 26 & 79 & & $\mathrm{~F}$ & $\mathrm{~F}$ & $\mathrm{~N}$ & $2017-10-2607: 38: 48$ \\
\hline 13 & 26 & 79 & & $\mathrm{~F}$ & $\mathrm{~F}$ & $\mathrm{~N}$ & $2017-10-2607: 38: 26$ \\
\hline 14 & 26 & 79 & & $\mathrm{~F}$ & $\mathrm{~F}$ & $\mathrm{~N}$ & $2017-10-2607: 38: 04$ \\
\hline 15 & 26 & 79 & & $\mathrm{~F}$ & $\mathrm{~F}$ & $\mathrm{~N}$ & $2017-10-2607: 37: 41$ \\
\hline 16 & 26 & 79 & & $\mathrm{~F}$ & $\mathrm{~F}$ & $\mathrm{~N}$ & $2017-10-2607: 37: 19$ \\
\hline
\end{tabular}

Fig 9: Live Monitoring the sensor parameters from IoT WebServer

Hello. wsn001

Logout Switch to Table View

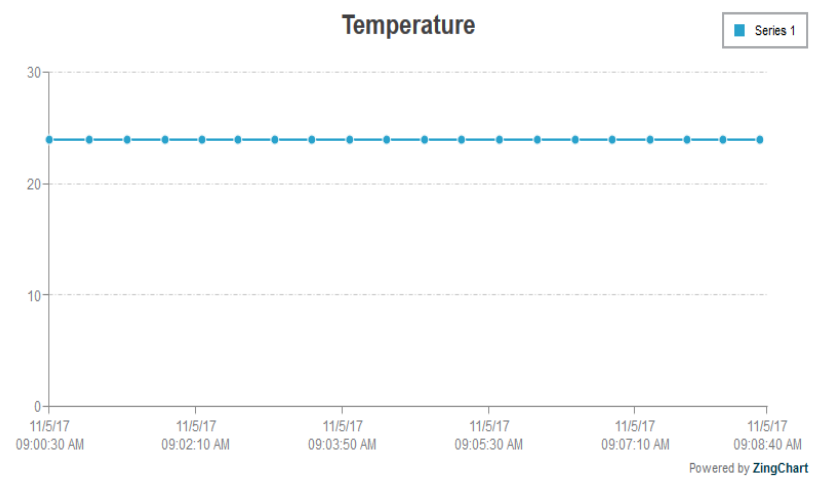

Fig10: Graphical Representation of Temperature

Humidity

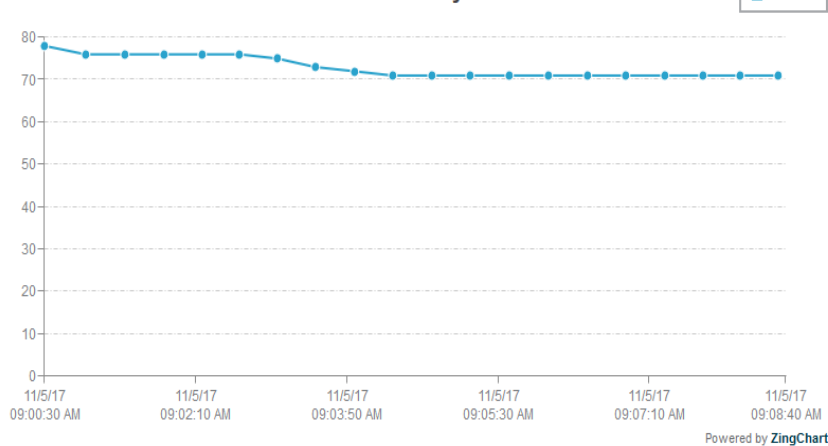

Fig11: Graphical Representation of Humidity

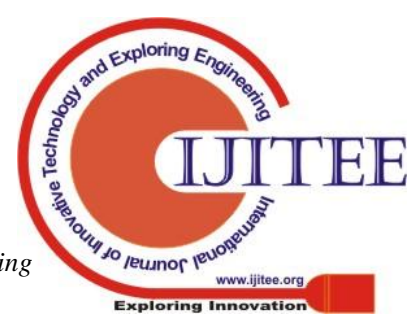




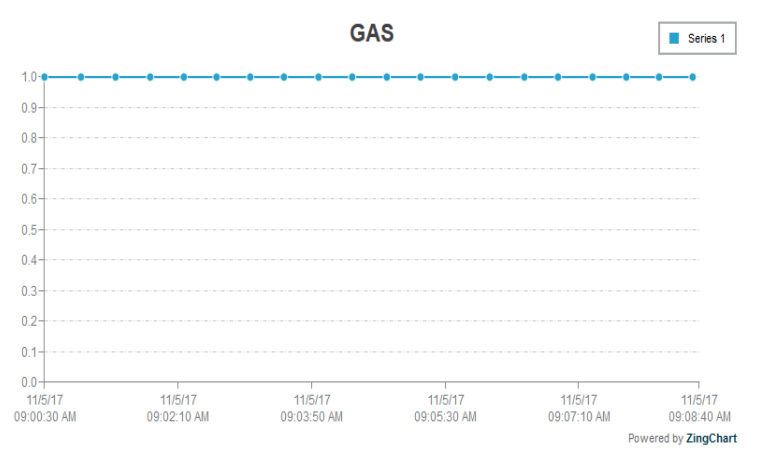

Fig12: Graphical Representation of Gas

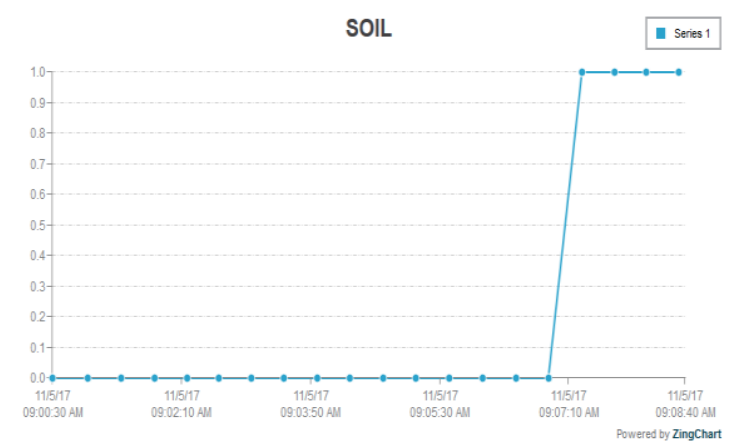

Fig13: Graphical Representation of Moisture sensor

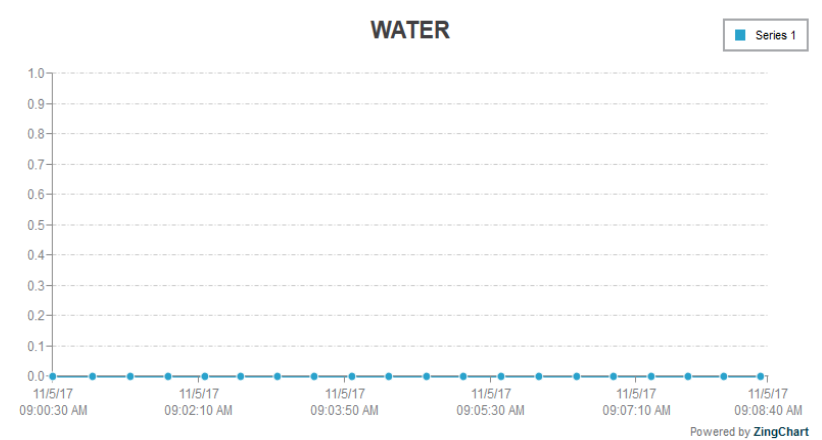

Fig14: Graphical representation of Water level

\section{VII . CONCLUSION}

This paper presents a platform for Wireless Sensor Network (WSN) which is reusable and best suits the wireless industrial automation in IoT environment with the application requirements, possible solutions for exploration, and the practical realization of a full-custom. The productivity of industrial systems can be improved with the IWSNs by providing proper awareness about the control, and integration of Industrial processes. In this manner the framework is actualized by shaping the system utilizing Zigbee and the sensor checking and Industrial computerization is done with the assistance of the sensor module and the process controller. This framework is truly dependable in situations where the remote correspondence is of fundamental concern. This framework is reasonable in ventures as the establishment is exceptionally basic with this Zigbee hubs and it is particularly proper for checking and controlling.

\section{REFERENCES}

1. Y. Fan, Y. Yin, L. Xu, Y. Zeng, and F. Wu, "IoT based smart rehabilitation system," IEEE Trans. Ind. Informat., vol. 10, no. 2, pp. 1568-1577, 2014.
2. W. He, G. Yan, and L. Xu, "Developing vehicular data cloud services in the IoT environment," IEEE Trans. Ind. Informat., vol. 10, no. 2, pp. 1587-1595, 2014.

3. S. Li, L. Xu, X. Wang, and J. Wang, "Integration of hybrid wireless networks in cloud services oriented enterprise information systems," Enterp. Inf. Syst., vol. 6, no. 2, pp. 165-187, 2012.

4. Q. Li, Z. Wang, W. Li, J. Li, C. Wang, and R. Du, "Applications integration in a hybrid cloud computing environment: Modelling and platform," Enterp. Inf. Syst., vol. 7, no. 3, pp. 237-271, 2013.

5. L. Wang, L. D. Xu, Z. Bi, and Y. Xu, "Data cleaning for RFID and WSN integration," IEEE Trans. Ind. Informat., vol. 10, no. 1, pp. 408- 418, Feb. 2014.

6. M. T. Lazarescu, "Design of a WSN platform for longterm environmental monitoring for IoT applications," IEEE J. Emerg. Sel. Topics Circuits Syst., vol. 3, no. 1, pp. 45-54, Mar. 2013.

7. L. Xu, "Introduction: Systems science in industrial sectors," Syst. Res. Behav. Sci., vol. 30, no. 3, pp. 211213, 2013.

8. Z. Pang et al., "Ecosystem analysis in the design of open platformbased in-home healthcare terminals towards the internet-of-things," in Proc. IEEE 15th Int. Conf. Adv. Commun. Technol. (ICACT), 2013, pp. 529-534. 